\title{
Zur Konstitution eines Silicats mit dem Anion $\left[\mathrm{Si}_{7} \mathrm{O}_{19}\right]^{10-}$
}

\author{
Von D. Hoebrel und W. WIEkeR
}

Mit 4 Abbildungen

Professor Günther Rienäcker zum 70. Geburtstage am 13. Mai 19 4 gewidmet

Inhaltsübersicht. Mit Hilfe chromatographischer und kinetischer Methoden und der Massenspektroskopie wurde im System $\mathrm{N}\left(\mathrm{CH}_{3}\right)_{4} \mathrm{OH}--\mathrm{SiO}_{2}-\mathrm{H}_{2} \mathrm{O}$ neben dem bekannten Tetramethylammonium-Doppelvierringsilicat ein bisher unbekanntes Tetramethylammo-

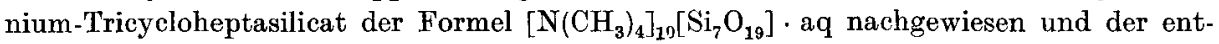
sprechende Trimethylsilylester $\left[\left(\mathrm{CH}_{3}\right)_{3} \mathrm{Si}_{10}\left(\mathrm{Si}_{7} \mathrm{O}_{19}\right]\right.$ isoliert.

On the Constitution of a Silicate with the Anion $\left[\mathrm{Si}_{7} \mathrm{O}_{19}\right]^{10-}$

Abstract. By means of chromatographic and kinetical methods and by mass spectroscopy the existence of a new silicate species in the system $\mathrm{N}\left(\mathrm{CH}_{3}\right)_{4} \mathrm{OH}-\mathrm{SiO}_{2}-\mathrm{H}_{2} \mathrm{O}$, the tetramethylammonium tricycloheptasilicate $\left[\mathrm{N}\left(\mathrm{CH}_{3}\right)_{4}\right]_{10}\left[\mathrm{Si}_{7} \mathrm{O}_{19}\right] \cdot a q$, has been revealed. The corresponding trimethylsilyl ester was isolated.

In einer früheren Arbeit ${ }^{1}$ ) wurde über die Konstitution eines Tetramethylammoniumsilicats der Zusammensetzung $1,0 \mathrm{~N}\left(\mathrm{CH}_{3}\right)_{4} \mathrm{OH} \cdot 1,0 \mathrm{SiO}_{2}$. $8,0-8,3 \mathrm{H}_{2} \mathrm{O}$ berichtet, das als Doppelvierringsilicat der Formel $\left[\mathrm{N}\left(\mathrm{CH}_{3}\right)_{4}\right]_{8}$ $\left[\mathrm{Si}_{8} \mathrm{O}_{20}\right] \cdot 69 \mathrm{H}_{2} \mathrm{O}$ identifiziert wurde. Weitere Untersuchungen am System $\mathrm{N}\left(\mathrm{CH}_{3}\right)_{4} \mathrm{OH}-\mathrm{SiO}_{2}-\mathrm{H}_{2} \mathrm{O}$ ergaben, daß außer den Doppelvierringsilicatanionen (DVR-Anionen) auch noch andere, bisher unbekannte Silicatanionentypen auftreten können.

Im folgenden wird über die Eigenschaften und die Konstitution eines dieser unbekannten Silicatanionen berichtet.

\section{1. Über die Herstellung von Tetramethylammoniumsilicaten (TMAS)}

Das Tetramethylammonium(TMA)-Doppelvierringsilicat der Zusammensetzung $1,0 \mathrm{~N}\left(\mathrm{CH}_{3}\right)_{4} \mathrm{OH} \cdot 1 \mathrm{SiO}_{2} \cdot 8 \mathrm{H}_{2} \mathrm{O}$, kristalliert in reinem Zustand aus wäßrigen Lösungen aus, in denen ein molares $\mathrm{N}\left(\mathrm{CH}_{3}\right)_{4} \mathrm{OH}: \mathrm{SiO}_{2}$-Verhältnis von $\mathrm{N}\left(\mathrm{CH}_{3}\right)_{4} \mathrm{OH}: \mathrm{SiO}_{2}=1: 1$ vorliegt. Wird das $\mathrm{N}\left(\mathrm{CH}_{3}\right)_{4} \mathrm{OH}: \mathrm{SiO}_{2}$ -

\footnotetext{
1) D. Hokbbel u. W. Wreker, Z. anorg. allg. Chem. 384, 43 (1971).
} 
Verhältnis der Lösungen $z$. B. auf $2: 1,3: 1$ usw. bis $8: 1$ vergrößert, so enthält die erste Kristallfraktion vorwiegend das TMA-Doppelvierringsilicat, daneben aber, mit steigendem $\mathrm{N}\left(\mathrm{CH}_{3}\right)_{4} \mathrm{OH}: \mathrm{SiO}_{2}$-Verhältnis der Lösung zunehmend ein neues TMA-Silicat. Dieses Silicat ließ sich bisher jedoch nicht rein darstellen, da es stets zusammen mit dem DVR-Silicat auskristallisiert. Die Analysen solcher gemischten Kristall-Fraktionen ergaben stets ein molares $\mathrm{N}\left(\mathrm{CH}_{3}\right)_{4} \mathrm{OH}: \mathrm{SiO}_{2}$-Verhältnis größer 1 .

In Tab. 1 sind z. B. die Analysenwerte von TMAS-Fraktionen, die durch fraktionierte Kristallisation einer Lösung mit einem $\mathrm{N}\left(\mathrm{CH}_{3}\right)_{4} \mathrm{OH}: \mathrm{SiO}_{2^{-}}$ Verhältnis von 3:1 erhalten wurden, und der entsprechenden Mutterlauge eingetragen.

Tabelle 1 Analysendaten der TMAS Fraktionen

\begin{tabular}{lrrl}
\hline & \multicolumn{3}{c}{ Molares $\mathrm{N}\left(\mathrm{CH}_{3}\right)_{4} \mathrm{OH}: \mathrm{SiO}_{2}-\mathrm{Verhältnis}$} \\
& Lösung & \multicolumn{1}{c}{ Kristalle } \\
\hline Ausgangslösung & $3: 1$ & $1,21: 1: 11 \mathrm{H}_{2} \mathrm{O}$ & 1. Kristallfrakt. \\
1. Mutterlauge & $7,4: 1$ & $1,43: 1: 10 \mathrm{H}_{2} \mathrm{O}$ & 2. Kristallfrakt. \\
2. Mutterlauge & $16,8: 1$ & &
\end{tabular}

Da, wie aus Tab. 1 hervorgeht, die kristallinen TMAS-Fraktionen mit einem kleineren $\mathrm{N}\left(\mathrm{CH}_{3}\right)_{4} \mathrm{OH}: \mathrm{SiO}_{2}$-Verhältnis aus dem in der Lösung vorgegebenen auskristallisieren, vergrößert sich demnach nach jeder Kristallisation das $\mathrm{N}\left(\mathrm{CH}_{3}\right)_{4} \mathrm{OH}: \mathrm{SiO}_{2}$-Verhältnis der zurückbleibenden Mutterlaugen.

Im folgenden soll vorrangig auf die Untersuchungen der in Tab.1 angegebenen 1. Kristallfraktion eingegangen werden.

\section{Papierchromatographische Untersuchungen}

Mit Hilfe papierchromatographischer Untersuchungen ${ }^{2}$ ) wurde festgestellt, daß sowohl in der 1 . Fraktion $\left(1,21 \mathrm{~N}\left(\mathrm{CH}_{3}\right)_{4} \mathrm{OH}: 1 \mathrm{SiO}_{2}\right)$ als auch in der 2. Kristallfraktion $\left(1,45 \mathrm{~N}\left(\mathrm{CH}_{3}\right)_{4} \mathrm{OH}: 1,0 \mathrm{SiO}_{2}\right)$ ein Gemisch aus zwei unterschiedlichen Silicatanionentypen enthalten ist. Aus den Chromatogrammen der Abb.1 geht deutlich hervor, daß ein Fleck des Gemisches $\left(\mathrm{R}_{\mathrm{F}}=0,76\right)$ dem bekannten TMA-Doppelvierringsilicat zuzuordnen ist. Der andere Fleck $\left(\mathrm{R}_{\mathrm{F}}=0,67\right)$ befindet sich in einer bisher nicht beobachteten Position zwischen der DVR- und Cyclohexa- bzw. Doppeldreiringsilicatposition. Aus den Fleckintensitäten ist zu entnehmen, daß in den TMAS-Fraktionen 1 und 2 unterschiedliche Mengenverhältnisse von DVRSilicatanionen zu unbekannten Silicatanionen vorliegen.

2) W. Wieker u. D. Hokbber, Z. anorg. allg. Chem. 366, 139 (1969). 


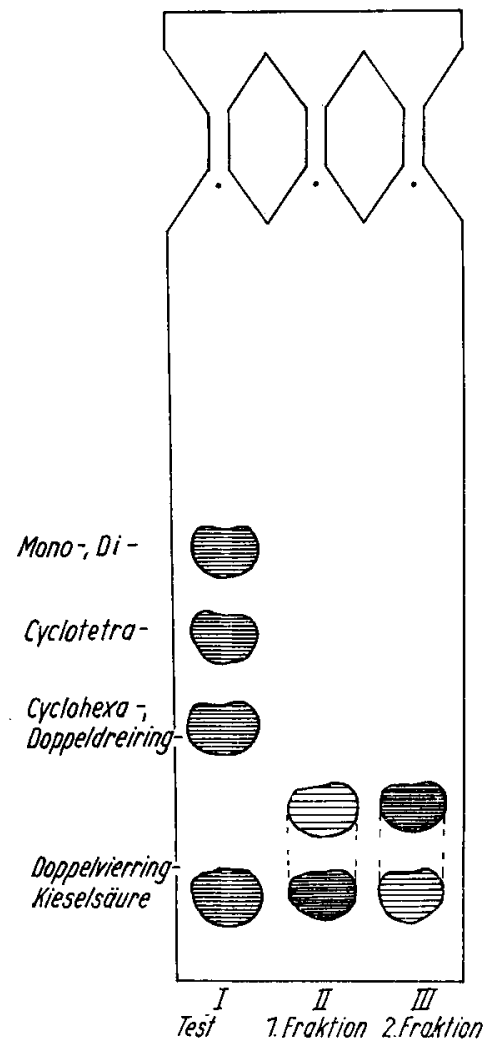

Abb.1. LI Papierchromatogramme der 1. und 2. TMAS-Kristallfraktion

Aus der Tatsache, daß sich die Laufgeschwindigkeiten der niedermolekularen Silicatanionen auf den Papierchromatogrammen mit zunehmender Anzahl der $\mathrm{SiO}_{4}$-Tetraeder im Anion vergrößern, ist aus der neuen Position zu schließen, daß das neue Silicatanion aus $7 \mathrm{SiO}_{4}$ Tetraedern aufgebaut sein müßte.

Nicht auszuschließen ist auch ein Silicatanion mit mehr als $8 \mathrm{SiO}_{4}$ Tetraedern, da bei Silicatanionen mit mehr als $8 \mathrm{SiO}_{4}$ Tetraedern wieder eine Abnahme der Laufgeschwindigkeiten auf den Chromatogrammen zu beobachten ist.

Zur Bestimmung des mengenmäßigen Anteils des neuen Anionentyps in den TMAS-Kristallfraktionen wurden quantitative papierchromatographische Analysen durchgeführt, nach denen in der 1. Kristallfraktion das TMAS mit Doppelvierringstruktur zu 57\% und das neue TMAS zu $43 \%$ enthalten ist, während in der 2. Kristallfraktion das Doppelvierringsilicat nur noch zu 32\% und das neue TMAS entsprechend zu $68 \%$ enthalten ist. Aus den Analysen läßt sich, da die Zusammensetzung des DVR-Silicats 
bekannt ist, die ungefähre Zusammensetzung des neuen TMAS berechnen. Für die 1. Fraktion wurde sie zu 1,49 N($\left(\mathrm{CH}_{3}\right)_{4} \mathrm{OH}: 1 \mathrm{SiO}_{2}: 15 \mathrm{H}_{2} \mathrm{O}$ und für die 2. Fraktion zu 1,63 N(CH$)_{4} \mathrm{OH}: 1 \mathrm{SiO}_{2}: 10 \mathrm{H}_{2} \mathrm{O}$ ermittelt. Auf Grund von GuINIER-Aufnahmen wurde nachgewiesen, daß in der 2. Fraktion kristallines $\mathrm{N}\left(\mathrm{CH}_{3}\right)_{4} \mathrm{OH} \cdot 5 \mathrm{H}_{2} \mathrm{O}$ enthalten ist. Daraus erklärt sich das höhere $\mathrm{N}\left(\mathrm{CH}_{3}\right)_{4} \mathrm{OH}: \mathrm{SiO}_{2}-$ Verhältnis der 2. Fraktion. Das molare $\mathrm{N}\left(\mathrm{CH}_{3}\right)_{4} \mathrm{OH}: \mathrm{SiO}_{2}-$ Verhältnis des neuen TMAS muß also um den Wert 1,49:1 liegen.

\section{Untersuchungen mit Hilfe der Molybdatmethode}

Durch kinetische Untersuchungen der TMAS Fraktionen sollte die nach den papierchromatographischen Untersuchungen noch offene Frage geklärt werden, ob in dem neuen TMAS-Anion 7 oder möglicherweise mehr als $8 \mathrm{SiO}_{4}$-Tetraeder enthalten sind.

Die Molybdatmethode ${ }^{3}$ ), die auf der Bestimmung der Bildungsgeschwindigkeit der gelben Silicimolybdänsäure aus Kieselsäuren und Molybdänsäuren beruht, ermöglicht durch Vergleich unbekannter Molybdatreaktionskurven mit definierten Molybdatreaktionskurven bekannter Silicatanionentypen Aussagen über die Silicatanionengröße. Abb. 2 zeigt einige Molybdatreaktionskurven von Silicaten mit bekannter Anionenkonstitution. Aus einem Vergleich des jeweiligen Endanstiegs der Molybdatreaktionskurven $1-5$ und den Reaktionszeiten mit Molybdänsäure geht hervor, daß mit zunehmender Anzahl von $\mathrm{SiO}_{4}$-Tetraedern im Silicatanion der Endanstieg der Molybdatreaktionskurven immer geringer wird.

In Abb. 2 sind auch die Molybdatreaktionskurven der 1. und 2. TMASFraktion mit eingezeichnet (D, C). Naturgemäß unterscheiden sich diese beiden Reaktionskurven voneinander, da die Fraktionen unterschiedlich zusammengesetzt sind und mit der Molybdatmethode immer nur die Summe der Reaktionsgeschwindigkeiten der einzelnen Silicatanionentypen gemessen wird. Beiden Molybdatreaktionskurven ist aber gemeinsam, daß sie im Vergleich zur Kurve des DVR-Silicatanions (Reaktionszeit 11,6 min) nach kürzeren Reaktionszeiten hin verschoben sind. Das bedeutet, da die Reaktionskurve der einen Silicatkomponente (DVR-Anion) bekannt ist, Kurve 5, daß die reine Molybdatreaktionskurve des neuen Silicatanions noch weiter nach kürzeren Reaktionszeiten $(<10,4 \mathrm{~min}$ ) verschoben sein muß.

Zwecks experimenteller Ermittlung der reinen Molybdatreaktionskurve des neuen Silicats wurde mit Hilfe der PC das neue Silicat vom DVR-Silicat getrennt, der betreffende Fleck eluiert und mit Hilfe der Molybdatmethode untersucht. Die gemessene Reaktionskurve zeigt Abb.2A. Auf Grund einer durch Papierrückstände bedingten leichten Trübung der zu fotometrierenden

3) E. Thilo, W. Wieker, u. H. Stade, Z. anorg. allg. Chem. 340, 261 (1965). 


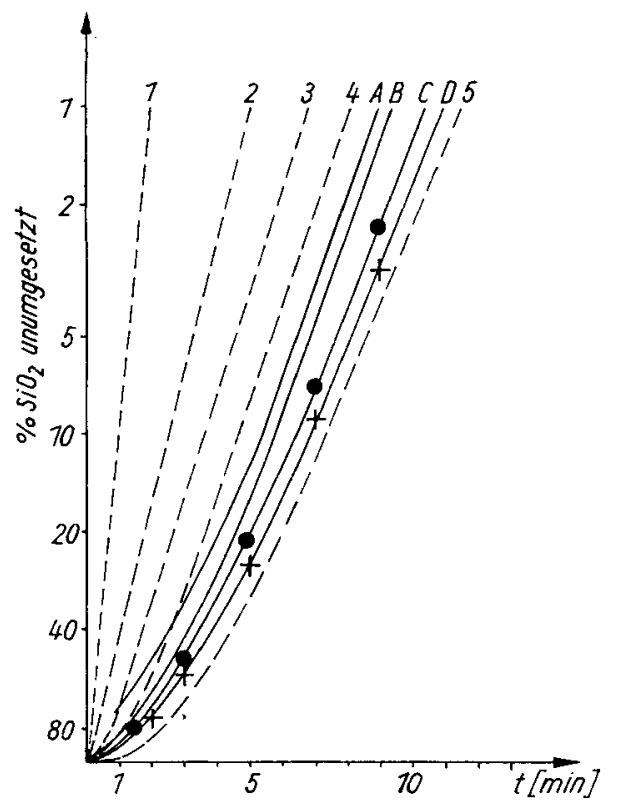

Abb. 2. Molybdatreaktionskurven der TMAS-Kristallfraktionen und von Testsubstanzen. 1 Mono-, 2 Di-, 3 Cyclotetra-, 4 Cyclohexasilicat, 5 TMA-Doppelvierringsilicat. A Originalkurve des extrahierten neuen Flecks $\left(\mathbf{R}_{\mathrm{F}}=0,67\right)$, B Korrigierte Kurve A, C TMAS 3:1, 2. Fraktion, D TMAS 3:1, 1. Fraktion

Lösung wird jedoch eine zu hohe Anfangsextinktion erhalten, so daß der Kurvenbeginn nicht im Ursprung liegt. Korrigiert man die gemessene Reaktionskurve so, daß der Ursprung bei $t=0$ liegt, so erhält man die Reaktionskurve B in Abb. 2. Zur Prüfung, ob diese experimentell ermittelte Molybdatreaktionskurve tatsächlich dem reinen neuen TMAS zukommt, wurden die Reaktionskurven ( $\mathbf{D}$ und $\mathbf{C}$ der Kristallfraktionen 1 und 2 auf der Grundlage der beiden Reaktionskurven $B$ und 5 der reinen Komponenten und des Verhältnisses des DVR-Silicats zum neuen TMAS in den Kristallfraktionen berechnet. Die berechneten Punkte, Abb. $2(+\bullet)$ stimmen gut mit den gemessenen Reaktionskurven $\mathrm{D}, \mathrm{C}$ der beiden Kristallfraktionen überein. Daraus ergibt sich, daß die Mo-Reaktionskurve $B$ tatsächlich dem reinen neuen. TMAS zukommt.

Aus der Reaktionszeit von 9,4 Minuten und dem im Vergleich zur Reaktionskurve des DVR-Anions steileren Endverlaufs der Kurve ist auf Grund. dieser Ausführungen zu entnehmen, daß die Variante mit mehr als $8 \mathrm{SiO}_{4^{-}}$ Tetraedern im Anion des neuen TMAS entfällt, so daß nur die Möglichkeit besteht, daß $7 \mathrm{SiO}_{4}$-Tetraeder das Anion des neuen TMAS aufbauen.

Wie diese $7 \mathrm{SiO}_{4}$-Tetraeder im Anion angeordnet sind, läßt sich aus den. 
bisherigen Untersuchungen nicht entnehmen. Zur Fortführung der Konstitutionsermittlung wurden die TMA-Silicate in die entsprechenden Trimethylsilylester überführt.

\section{Trimethylsilylierung der 1. TMAS-Fraktion}

Nach der von GöTz und. Mitarb. ${ }^{4}$ ) modifizierten Trimethylsilylierungsmethode von LENTZ ${ }^{5}$ ) wurde das TMAS-Gemisch der summarischen Zusammensetzung 1,21 $\mathrm{N}\left(\mathrm{CH}_{3}\right)_{4} \mathrm{OH}: 1,0 \mathrm{SiO}_{2}: 11 \mathrm{H}_{2} \mathrm{O}$ verestert. Beim Einengen der Hexamethyldisiloxanphase fielen farblose Kristalle aus, die mit Hilfe der Dünnschichtchromatographie ${ }^{6}$ ) als Ester des Doppelvierring-

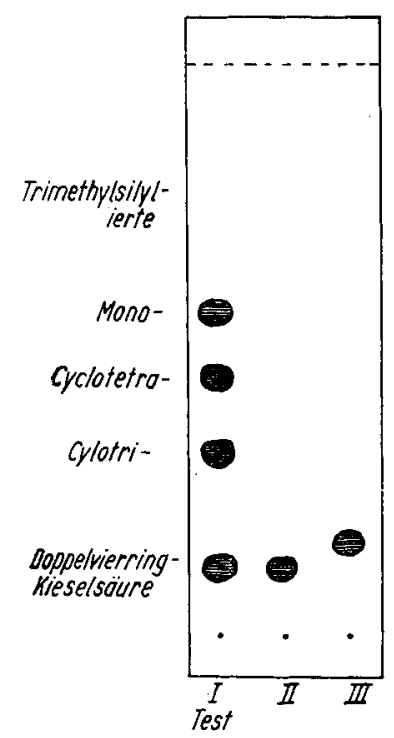

Abb.3. Dünnschichtchromatogramme des trimethylsilylierten Doppelvierringsilicats (Bahn II) und des neuen trimethylsilylierten Silicats (Bahn III)

silicats identifiziert wurden. Abb.3, Bahn II. Aus der Restlösung bildeten sich langsam gut ausgebildete Kristalle, die sich in ihrer dünnschichtchromatographischen Position deutlich von dem DVR-Ester durch eine größere Laufgeschwindigkeit unterscheiden. Abb.3, III. Von den beiden verschiedenen Estern wurden etwa gleiche Mengen erhalten. Bezogen auf das eingesetzte Silicat wurden die beiden Ester in einer Ausbeute von $80 \%$ erhalten.

Wie zu erwarten, bildete sich also neben dem DVR-Ester in 80proz. Ausbeute ein neuer, bisher unbekannter Ester, der aus dem neuen TMA-Silicat entstanden sein müßte.

$\left.{ }^{4}\right)$ J. Göтz u. C. R. Masson, J. chem. Soc. [London] Secr. A 1970, 2683.

$\left.{ }^{5}\right)$ C. W. Lentz, Inorg. Chem. [Washington] 3, 574 (1964).

6) D. Hoebbei u. W. Wieker, Z. anorg. allg. Chem. 405, 163 (1974). 
Zur Ủberprüfung, ob sämtliche $\equiv$ SiOH-Gruppen im unbekannten Ester silyliert waren, wurden Protonenresonanzspektren des Esters in $\mathrm{CCl}_{4}$ bei $60 \mathrm{MHz}$ aufgenommen. Die Spektren zeigten keine $\equiv \mathrm{SiOH}-$ Protonenresonanzen, so daß eine vollständige Silylierung anzunehmen ist.

Nach Göтz ${ }^{7}$ ) lassen sich die Molekulargewichte silylierter Kieselsäuren massenspektrometrisch sehr genau bestimmen, da die Fragmentierung vorwiegend unter Abspaltung einer $-\mathrm{CH}_{3}$-Gruppe erfolgt. Die Massenzahl des intensitätsstärksten Peaks entspricht dann dem um 15 Masseeinheiten erniedrigten Molekulargewicht M des Esters. Der M-15 Peak des neuen Esters wurde mit $\mathrm{m} / \mathrm{e}=1215$ bestimmt, d. h. das Molekulargewicht des Esters beträgt $\mathrm{M}=1230$.

Die Ergebnisse der bisherigen Untersuchungen des neuen TMAS und des entsprechenden Trimethylsilylesters sind in Tab. 2 zusammengefaßt.

Auf Grund dieser Daten eregeben sich für die Konstitution des Esters bzw. des neuen TMAS mehrere isomere tricyclische Formen, die jeweils gleiche Zusammensetzung und auch gleiche Molekulargewichte aufweisen. Abb. 4 zeigt in schematischer Darstellung einige der möglichen Konstitutionen. Zwischen diesen Konstitutionen ist mit den hier verwendeten Untersuchungsmethoden nicht zu unterscheiden. Die Konstitution (1) kann man

Tabelle 2 Analysenergebnisse

\begin{tabular}{|c|c|c|}
\hline & Ermittelte Daten & $\begin{array}{l}\text { Daten der vorge- } \\
\text { schlag. Konstitutionen }\end{array}$ \\
\hline $\begin{array}{l}\text { Anzahl d. Si-Atome } \\
\text { im Anion }\end{array}$ & 7 & 7 \\
\hline $\begin{array}{l}\text { Molares } \mathrm{N}\left(\mathrm{CH}_{3}\right)_{4} \mathrm{OH} \text { : } \\
\mathrm{SiO}_{2} \text {-Verhältnis }\end{array}$ & $\leq 1,49: 1$ & $1,42: 1$ \\
\hline Analyse d. Esters & $7,46 \% \mathrm{H} ; 30,2 \% \mathrm{C}$ & $7,32 \% \mathrm{H} ; 29,3 \% \mathrm{C}$ \\
\hline $\begin{array}{l}\text { Molekulargewicht } \\
\text { des Esters }\end{array}$ & 1230 & 1230 \\
\hline
\end{tabular}

sich aus einem Doppelvierringsilicatanion entstanden denken, durch Verlust eines $\mathrm{SiO}_{4}$-Tetraeders. Durch Neuorientierung zweier Bindungen am $\mathrm{SiO}_{4}^{*}$ Tetraeder der Konstitution (1) würden sich die Konstitutionen (2) und (3) bilden. Auch die Konstitution (4) steht nicht im Widerspruch zu den Untersuchungsergebnissen.

Da in dem TMAS mit einem $\mathrm{N}\left(\mathrm{CH}_{3}\right)_{4} \mathrm{OH}: \mathrm{SiO}_{2}-V e r h a ̈ l t n i s$ von $1: 1$ und auch in seinen konzentrierten wäßrigen Lösungen nur bzw. hauptsächlich die beständigen DVR-Anionen enthalten sind und mit steigendem

7) F. F. H. Wu, J. Götz, W. D. Jamieson u. C. R. Masson, J. Chromatogr. [Amsterdam] 48, 515 (1970).

18 Z. anorg. allg. Chemie. Bd. 405. 

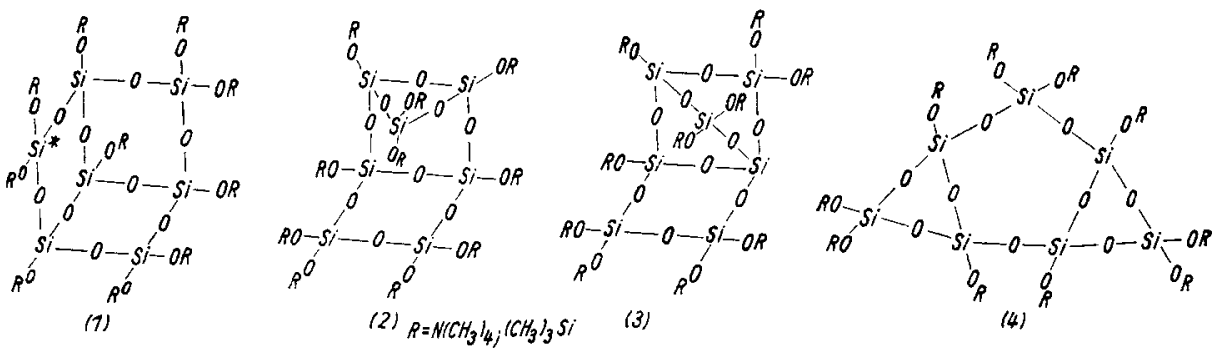

Abb. 4. Mögliche Konstitutionsschemata des neuen TMA-Silicats

$\mathrm{N}\left(\mathrm{CH}_{3}\right)_{4} \mathrm{OH}: \mathrm{SiO}_{2}$-Verhältnis in zunehmendem Maße das neue TMA-Silicatanion zu ungunsten der Bildung von DVR-Silicatanionen entsteht, ist anzunehmen, daß das TMAS-Anion aus dem vorhandenen DVR-Anion gebildet wird. Vom Standpunkt der bisherigen Untersuchungen ist daher den Konstitutionen (1) bis (3) der Vorzug zu geben, wobei die Ausbildung der Konstitution (3) wenig wahrscheinlich ist, da in ihr ein Si-Atom vorliegen müßte, das vier $\equiv \equiv \mathrm{Si}-\mathrm{O}-\mathrm{Si} \equiv$-Bindungen mit anderen $\mathrm{SiO}_{4}$-Tetraedern eingeht.

Das bisher unbekannte Tetramethylammoniumsilicat der Zusammensetzung $1,42 \mathrm{~N}\left(\mathrm{CH}_{3}\right)_{1} \mathrm{OH} \cdot 1 \mathrm{SiO}_{2} \cdot \mathrm{aq}$ ist allgemein als Tricycloheptasilicat der Formel

$$
\left[\mathrm{N}\left(\mathrm{CH}_{3}\right)_{4}\right]_{10}\left[\mathrm{Si}_{7} \mathrm{O}_{19}\right] \cdot \mathrm{aq}
$$

und der Trimethylsilylester entsprechend als

$$
\left[\left(\mathrm{CH}_{3}\right)_{3} \mathrm{Si}_{10}\left[\mathrm{Si}_{7} \mathrm{O}_{19}\right]\right.
$$

$\mathrm{zu}$ formulieren.

In der polycyclischen Methylsiloxanreihe wurde von SсотT $^{8}$ ) eine der hier untersuchten Verbindung entsprechende Verbindung $\left(\mathrm{CH}_{3}\right)_{10} \mathrm{Si}_{7} \mathrm{O}_{9}$ aufgefunden, für die jedoch auch noch keine konkrete Zuordnung zu einer bestimmten Konstitution erfolgte.

Den Herren Dr. P. Fraxke und Dr. H. A. Otro, Zentralinstitut für Molekularbiologie der $\mathrm{AdW}$ der $\mathrm{DDR}$, möchten wir für die massenspektroskopischen Untersuchungen der Kieselsäureester danken.

Berlin-Adlersh of, Zentralinstitut für Anorganische Chemie der Akademie der Wissenschaften der DDR.

Bei der Redaktion eingegangen am 30. August 1973.

Anschr. d. Verf.: Dr. D. Hoebeel und Prof. Dr. habil. W. WIEker

Zentralinst. f. Anorg. Chemie d. AdW der DDR

DDR-1199 Berlin-Adlershof, Rudower Chaussee 5

8) D. W. Scotт, J. Amer, chem. Soc. 68, 356 (1946). 